\title{
The Nordic Aortic Valve Intervention (NOTION) trial comparing transcatheter versus surgical valve implantation: study protocol for a randomised controlled trial
}

\author{
Hans Gustav Thyregod ${ }^{1 *}$, Lars Søndergaard², Nikolaj Ihlemann², Olaf Franzen², Lars Willy Andersen², \\ Peter Bo Hansen ${ }^{3}$, Peter Skov Olsen ${ }^{1}$, Henrik Nissen ${ }^{4}$, Per Winkel ${ }^{5}$, Christian Gluud ${ }^{5}$ and \\ Daniel Andreas Steinbrüchel ${ }^{1}$
}

\begin{abstract}
Background: Degenerative aortic valve (AV) stenosis is the most prevalent heart valve disease in the western world. Surgical aortic valve replacement (SAVR) has until recently been the standard of treatment for patients with severe AV stenosis. Whether transcatheter aortic valve implantation (TAVI) can be offered with improved safety and similar effectiveness in a population including low-risk patients has yet to be examined in a randomised setting.

Methods/Design: This randomised clinical trial will evaluate the benefits and risks of TAVI using the transarterial CoreValve System (Medtronic Inc., Minneapolis, MN, USA) (intervention group) compared with SAVR (control group) in patients with severe degenerative AV stenosis. Randomisation ratio is 1:1, enrolling a total of 280 patients aged 70 years or older without significant coronary artery disease and with a low, moderate, or high surgical risk profile. Trial outcomes include a primary composite outcome of myocardial infarction, stroke, or all-cause mortality within the first year after intervention (expected rates 5\% for TAVI, 15\% for SAVR). Exploratory safety outcomes include procedure complications, valve re-intervention, and cardiovascular death, as well as cardiac, cerebral, pulmonary, renal, and vascular complications. Exploratory efficacy outcomes include New York Heart Association functional status, quality of life, and valve prosthesis and cardiac performance. Enrolment began in December 2009, and 269 patients have been enrolled up to December 2012.
\end{abstract}

Discussion: The trial is designed to evaluate the performance of TAVI in comparison with SAVR. The trial results may influence the choice of treatment modality for patients with severe degenerative AV stenosis.

Trial registration: ClinicalTrials.gov: NCT01057173

Keywords: Aortic valve stenosis, Aortic valve prosthesis, Transcatheter aortic valve implantation, Surgical aortic valve replacement, Randomised clinical trial design

\footnotetext{
* Correspondence: hgthyregod@gmail.com

'Department of Cardiothoracic Surgery, The Heart Centre, Rigshospitalet, Copenhagen University Hospital, Blegdamsvej 9, DK 2100, Copenhagen, Denmark

Full list of author information is available at the end of the article
} 


\section{Background}

Degenerative aortic valve (AV) stenosis is the most prevalent heart valve disease in the western world, affecting 2 to $7 \%$ of people older than 65 years of age. Both the incidence and prevalence are expected to rise due to the general increase in life expectancy [1,2]. The disease has a chronic course and carries a poor prognosis after onset of symptoms [3]. Medical therapy offers sparse symptomatic relief, and symptomatic patients have a 2-year survival of approximately 50\%. Surgical aortic valve replacement (SAVR) is an effective treatment, and complication rates and prosthesis durability are well known. The surgical procedure carries a low operative risk in younger patients without any significant co-morbidity (30-day mortality 3 to $5 \%$ for age $<70$ years). This risk increases substantially with increasing age, reduced left ventricular function, and other comorbidities (30-day mortality 5 to $15 \%$ ) [4,5]. Presumably because of this increase, almost one-third of patients referred for valve intervention at the time of trial design did not receive valve replacement and were continued on medical therapy [6]. A less invasive treatment option would therefore be attractive.

Different operative risk calculators in cardiac surgery (the Society of Thoracic Surgery Predicted Risk of Mortality (STS) score [7] and the European System for Cardiac Operative Risk Evaluation (EuroSCORE) [8]) have been developed to identify the high-risk surgical patient, but these are inadequate and generally overestimate the operative mortality in SAVR. A new EuroSCORE II has been developed and is being considered for implementation [9]. Data from randomised clinical trials are needed to improve treatment decision-making.

In recent years a new treatment option has become available for patients considered at high risk for SAVR. Transcatheter aortic valve implantation (TAVI) was originally developed in 1992 by Andersen and coworkers and was clinically introduced in 2002 by Cribier and coworkers as a minimally invasive treatment for patients considered ineligible for valve surgery $[10,11]$. The term TAVI comprises different valve prosthesis types, techniques, and approaches to the stenosed valve. Originally developed as a transvenous transseptal septal technique, the procedure is currently performed on the beating heart either antegrade transapically through a small left anterior thoracotomy or retrograde through the arterial system using either the femoral, subclavian, or carotid artery, or with the direct transaortic approach [12,13]. The artery can be surgically exposed or punctured. The two most widely used TAVI systems, both Conformité Européenne (European Conformity) marked, are the Edwards SAPIEN with a balloon-expandable bioprosthesis (Edwards Lifescience Inc., Irvine, CA, USA) and the CoreValve System with a self-expandable bioprosthesis
(Medtronic Inc., Minneapolis, MN, USA). The former has also been Food and Drug Administration approved in the United States.

Postoperative complications related to SAVR stem from sternotomy, cardiopulmonary bypass, aortic crossclamping, and cardioplegic cardiac arrest. In TAVI many of the SAVR complications are potentially avoided, but others are potentially exacerbated such as neurological lesions (2 to $11 \%$ during the first year) $[14,15]$. Retrograde catheter passage in the aortic arch and ascending aorta, and native valve pre-dilatation - causing calcified and often ulcerated leaflets to fracture, exposing thrombogenic endothelial lesions to the circulation could generate atherosclerotic emboli. At the same time, TAVI-specific complications have been encountered including haemodynamic instability and arrhythmia during implantation, prosthesis misplacement, and incomplete frame expansion (leading to prosthesis embolisation, valvular and paravalvular leakage, and valve-in-valve implantation, or conversion to open surgery), aortic and ventricular perforations, and arterial access lesions [16]. Furthermore, the CoreValve System has a high frequency of conduction abnormalities (atrioventricular and left bundle branch block) requiring a permanent pacemaker in 20 to $30 \%$ of patients, presumably because of septal compression [17]. Since operator experience has grown and implantation systems have improved and become smaller, many of these complications have become less frequent.

National and international registries have documented good short-term and mid-term safety results after TAVI in patients considered at high risk for surgery with a 95\% procedure success rate, and with 30-day mortality, stroke, and myocardial infarction rates of 5 to $12 \%, 2$ to $10 \%$, and 1 to $4 \%$, respectively, combined with excellent sustained prosthesis function, and clinical improvements [15,16,18-20]. Long-term results are lacking, but no reports of fractured prosthetic stents or frames have been published. Anecdotal reports of calcified TAVI prostheses after 5 years have been published [21].

Results after 1 and 2 years from the randomised trial (PARTNER Trial) comparing the Edwards SAPIEN prosthesis with standard medical therapy in patients considered to have prohibitive high surgical risk (Cohort B), and with SAVR in patients with high surgical risk (Cohort A) have been published [22-25]. For Cohort B an all-cause mortality reduction within the first year of $20 \%$ was observed (TAVI vs. medical therapy, $30.7 \%$ vs. 50.7\%; $P<0.001$ ), and for Cohort A non-inferiority for all-cause death after 1 year was proven (TAVI vs. SAVR, $24.2 \%$ vs. $26.8 \% ; P=0.44)$. Complication rates were significantly different, with more strokes and vascular injuries in the TAVI group but more major bleedings and more new-onset atrial fibrillation in the SAVR group. Another randomised trial using the Edwards SAPIEN 
system including low-risk patients has been reported. This trial was prematurely terminated after including 70 patients because of a nonsignificant excess of events in the TAVI group [26].

No randomised trials comparing TAVI versus SAVR in patients with low, moderate, and high operative risk and using the CoreValve System have been reported. Typically, high-risk patients considered for TAVI in current clinical practice have a logistic EuroSCORE $>20 \%$ and STS score $>10 \%$ predicted 30 -day operative mortality. Low-risk patients could have a logistic EuroSCORE of $3 \%$ and a STS score of $1 \%$. Patient preferences, the less invasive technique, and unclear indications could promote off-label use of TAVI, as has been seen in percutaneous coronary intervention. A randomised clinical trial can examine this new treatment option more extensively and generate evidence needed to broaden, optimise, and guide treatment for patients with severe degenerative AV stenosis [27].

\section{Methods/Design}

\section{Trial design and objectives}

The Nordic Aortic Valve Intervention (NOTION) trial is a multicentre, randomised clinical trial without blinding comparing TAVI using the CoreValve System versus SAVR in the treatment of severe AV stenosis designed during 2008/09 and launched in December 2009. The primary objective is to evaluate the safety and effectiveness of TAVI using the CoreValve System compared with SAVR using cardiopulmonary bypass.

The regional ethics committee has approved the trial at each clinical site, and all patients will provide written informed consent. The trial is conducted according to the Declaration of Helsinki. The organisational and coordinating site is The Heart Centre, Rigshospitalet, Copenhagen University Hospital, Copenhagen, Denmark, and the regional Research Ethics Committee in The Capital Region of Denmark approved the protocol. Other clinical sites are Odense University Hospital, Odense, Denmark, and Sahlgrenska University Hospital, Gothenburg, Sweden. The trial is registered at ClinicalTrials.gov (NCT01057173).

The trial is investigator initiated, designed, and conducted with no sponsor involvement. The trial has a steering committee, an independent event adjudication committee, and an external independent data monitoring and safety committee (DMSC). The DMSC is composed of an interventional cardiologist, a cardiac surgeon, a neurologist, and a statistician.

\section{Device and procedures}

The CoreValve System is a TAVI system designed to treat AV stenosis [20]. The procedure can be done on the beating heart with a retrograde arterial access. The third-generation system used for this trial includes a valve bioprosthesis consisting of a porcine pericardial trileaflet valve mounted in a nitinol self-expandable frame (frame diameter size $23,26,29$, or $31 \mathrm{~mm}$ covering an aortic annulus diameter from 18 to $29 \mathrm{~mm}$, length 52 to $55 \mathrm{~mm}$; Figure 1), and an 18 French (6 $\mathrm{mm}$ ) delivery system that enables implantation of the prosthesis. The TAVI procedure is performed under local or general anaesthesia in the cardiac catheterisation laboratory, either percutaneously via the femoral artery or after surgical cut-down of the subclavian artery. Before implantation, the native AV is pre-dilated with a balloon valvulotomy during rapid ventricular pacing to ensure a stable position of the balloon. The prosthesis is crimped in the delivery catheter. During deployment, the prosthetic frame will expand to its preformed shape and lodge itself in the left ventricular outflow tract, the aortic annulus, and the ascending aorta. The prosthesis can be retrieved until fully deployed, and an intervention catheter can pass through the frame to the coronary ostia. The access vessel is closed either with a percutaneous suture system (ProStar XL or Perclose ProGlide; Abbott Vascular, Inc., Menlo Park, CA, USA) or surgically. Before implantation a temporary pacemaker lead is placed in the right ventricle, used for rapid pacing and in case of conduction abnormalities. Correct prosthesis placement during and after deployment is verified by fluoroscopy and echocardiography. Before the procedure, patients receive a loading dose of $300 \mathrm{mg}$ clopidogrel. During the procedure, intravenous antibiotics are given before skin puncture, and 5,000 units heparin are given after vascular access has been established. After the procedure, patients receive $75 \mathrm{mg}$ clopidogrel daily for 3 months as well as $75 \mathrm{mg}$ acetyl salicylic acid lifelong. If vitamin $\mathrm{K}$ antagonist therapy is indicated, this is added to the above treatment. Other cardiac medications are prescribed according to local cardiologic guidelines.

SAVR is performed using standard surgical techniques and general anaesthesia. The procedure includes a complete median sternotomy, cardiopulmonary bypass in normothermia, aortic cross-clamping, and cold blood cardioplegic cardiac arrest. The native valve is excised and the annulus is carefully decalcified and irrigated before a bioprosthesis is sutured in place. The choice of type and size of the prosthesis is at the discretion of the surgeon. Radiofrequency ablation will be performed for chronic or intermittent atrial fibrillation. Postoperative antiplatelet and other cardiac medical therapy is similar to the TAVI group.

After the procedure, both patient groups will be admitted to a specialised cardiac intensive care unit at least overnight, and TAVI patients will thereafter be transferred to the cardiology ward and SAVR patients to the cardiac surgery ward. 

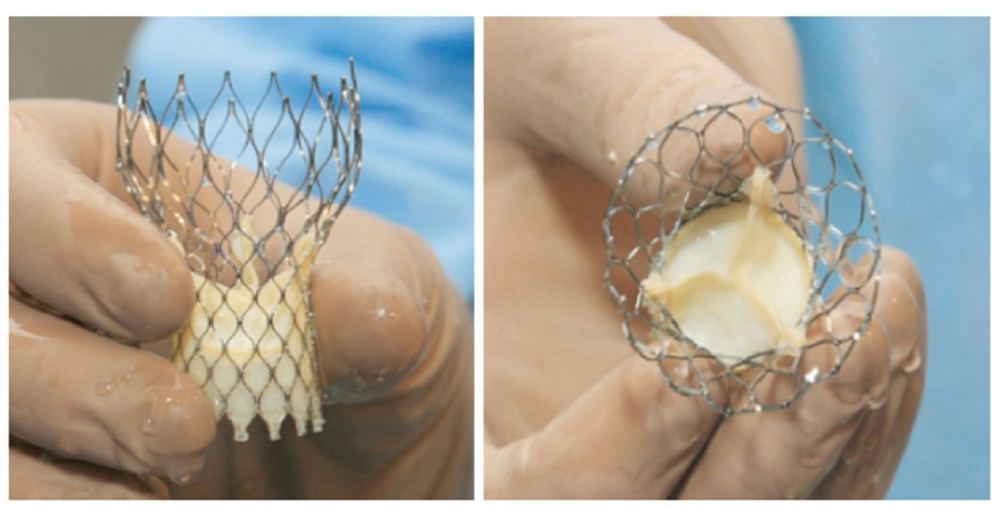

Figure 1 The transcatheter aortic valve implantation bioprosthesis. The third-generation porcine pericardial trileaflet valve mounted in a self-expanding nitinol support frame (CoreValve System; Medtronic Inc., Minneapolis, MN, USA). TAVI, transcatheter aortic valve implantation.

\section{Patient population}

Eligible patients include those aged 70 years or older with severe degenerative AV stenosis with symptoms or without symptoms but with left ventricular systolic dysfunction and/or hypertrophy (see Table 1 for all inclusion and exclusion criteria). Patients must be suitable for both TAVI and SAVR according to a cardiac surgeon, an interventionist, and an echocardiographer at a multidisciplinary conference. Inclusion criteria follow general surgical indications and the TAVI system manufacturer's guidelines for severe AV stenosis. Patients with previous heart surgery, other significant valve disease, or coronary artery disease requiring revascularisation at the time of referral are excluded. Patients with a stroke or transient ischemic attack within the previous 30 days or an acute coronary syndrome within the previous year are also excluded.

\section{Patient screening, randomisation, and follow-up}

All patients consecutively referred for SAVR at each study centre (all tertiary university hospitals) have been screened since December 2009 for eligibility at a multidisciplinary conference (Figure 2). Patients determined as ineligible are recorded for registries and follow-up. Potential eligible patients are interviewed and informed of trial risks and objectives before obtaining informed consent. Transthoracic echocardiography (transoesophageal studies are done if appropriate), coronary, aortic, and femoro-iliac angiograms, lung function tests, and standard laboratory blood samples are obtained in order to determine eligibility. Electrocardiogram (ECG)-gated high-resolution computed tomography studies can be used to evaluate the aortic annulus and root, and nonECG-gated studies can further evaluate the arterial system.

The trial will be conducted at three sites in Scandinavia and will enrol up to 280 patients. Randomisation will be 1:1 with approximately 140 patients in each group (TAVI and SAVR) and stratified according to age (70 to 74 years or $\geq 75$ years), coronary status (coronary artery disease not requiring revascularisation or normal), and trial site. Randomisation is central at the Copenhagen Trial Unit, which has generated the allocation sequence in permuted blocks with unknown block size for the investigators.

Patients undergo the assigned treatment with either TAVI using the CoreValve System or SAVR. Clinical follow-up with outcome measure recording, blood samples, and ECG occurs at pre-discharge and at 1, 3, 6, 12 months, and annually for a minimum of 5 years. Transthoracic echocardiography studies are performed before discharge and after 3 and 12 months, and annually. Patients will furthermore be followed in external electronic medical records and retrieved medical records for hospital admissions, the Danish National Hospital Registry [28], the Civil Registration System [29], and the Registry of Causes of Death [30].

\section{Outcomes}

The primary outcome is the composite of myocardial infarction, stroke, or all-cause mortality within the first 12 months (Table 2). Formal neurological evaluations and computed tomography cerebral studies will only be performed when clinically indicated and not routinely. ECGs and coronary markers will be evaluated routinely the first 3 days after the intervention and when indicated.

Exploratory safety outcomes at 30 days and 12 months include all-cause mortality; cardiovascular mortality; cardiac and prosthetic AV complications (myocardial infarction; new-onset arrhythmias and conduction abnormalities; prosthetic AV re-intervention, and endocarditis); stroke (ischaemic and haemorrhagic). Exploratory safety outcomes at 30 days include pulmonary, renal, accessrelated vascular and bleeding complications. Exploratory safety outcomes at 12 months include number of days hospitalised. 


\section{Table 1 Inclusion and exclusion criteria}

\begin{tabular}{|c|c|}
\hline & clusion criteria \\
\hline & $\begin{array}{l}\text { Severe degenerative AV stenosis (calcified AV, effective orifice area }<1 \\
\mathrm{~cm}^{2} \text { or indexed for body surface area }<0.6 \mathrm{~cm}^{2} / \mathrm{m}^{2} \text {, mean AV } \\
\text { gradient }>40 \mathrm{mmHg} \text {, or AV peak systolic velocity }>4.0 \mathrm{~m} / \mathrm{second} \text { ), } \\
\text { and }\end{array}$ \\
\hline & $\begin{array}{l}\text { Symptomatic (dyspnoea } \geq \text { NYHA class II, angina pectoris, or syncope), } \\
\text { or }\end{array}$ \\
\hline & Asymptomatic with one or more of the following: \\
\hline & - Left ventricle posterior wall thickness $\geq 17 \mathrm{~mm}$ \\
\hline & - Left ventricular ejection fraction $<60 \%$ but $\geq 20 \%$ \\
\hline & - Atrial fibrillation \\
\hline & Age $\geq 70$ years \\
\hline & $\begin{array}{l}\text { Candidate (clinical and anatomical) for both TAVI and SAVR (as } \\
\text { specified by prosthesis manufacture's guidelines) judged by a } \\
\text { multidisciplinary conference }\end{array}$ \\
\hline & Expected to survive $\geq 1$ year after intervention \\
\hline & Able to provide written informed consent \\
\hline & «clusion criteria \\
\hline & Isolated AV insufficiency \\
\hline & Other significant cardiac valve or septal diseases \\
\hline & Coronary artery comorbidity requiring revascularisation ( $\mathrm{PCl}$ or $\mathrm{CABG}$ ) \\
\hline & Intracardiac lesion (thrombus, tumour, vegetation) \\
\hline & Previous open cardiac surgery \\
\hline & Myocardial infarction or $\mathrm{PCl}$ within the last year \\
\hline & Stroke or transient ischemic attack within the last 30 days \\
\hline & Renal insufficiency requiring haemodialysis \\
\hline & $\begin{array}{l}\text { Pulmonary insufficiency (FEV1 or diffusion capacity }<40 \% \text { of } \\
\text { expected) }\end{array}$ \\
\hline & Active infectious disease requiring antibiotics \\
\hline & $\begin{array}{l}\text { Emergency intervention (within } 24 \text { hours after the indication for } \\
\text { intervention has been made) }\end{array}$ \\
\hline & $\begin{array}{l}\text { Unstable pre-interventional condition requiring inotropic support or } \\
\text { mechanical cardiac assistance }\end{array}$ \\
\hline & $\begin{array}{l}\text { A known hypersensitivity or contraindication to nitinol, heparin, } \\
\text { clopidogrel, acetyl salicylic acid, or contrast material }\end{array}$ \\
\hline & $\begin{array}{l}\text { Currently participating in an investigational drug or another device } \\
\text { study }\end{array}$ \\
\hline
\end{tabular}

$\mathrm{AV}$, aortic valve; $\mathrm{CABG}$, coronary artery bypass grafting; FEV1, forced expiratory volume in 1 second; NYHA, New York Heart Association; PCI, percutaneous coronary intervention; SAVR, surgical aortic valve replacement; TAVI, transcatheter aortic valve implantation.

Exploratory patient-oriented and prosthetic AV performance outcomes at 3 and 12 months include New York Heart Association functional class, quality of life (Short-form health survey with 36 questions questionnaire), and prosthetic AV opening area, peak and mean pressure gradients, prosthetic-patient mismatch, and regurgitation, as well as left ventricular function.

All outcomes will be assessed and recorded before discharge and at all follow-up visits.
Definitions of outcomes are adopted from the generally accepted Valve Academic Research Consortium consensus report for TAVI clinical trials $[31,32]$.

Evaluation of the primary outcome measure by the independent event adjudication committee will be blinded to the intervention performed. The blinding consists of removal of all information related to the type of intervention and prosthesis from collected patient records. The evaluation of exploratory outcomes, except for quality of life data, is difficult to blind since procedure and prosthesis types can be identified.

All required trial data and outcomes will be collected on standardised patient report forms and transferred to a central database for storage. The principal investigators at the organising trial site will manage and prepare data for publication, and an external independent statistician will perform the statistical analysis.

\section{Sample size calculation}

The trial is designed as a randomised superiority trial with the alternative hypothesis that TAVI is better than SAVR regarding the effect on the primary composite outcome consisting of myocardial infarction, stroke, or all-cause mortality after 1 year of follow-up. As the primary outcome measure is a binary variable, the sample size may be estimated using a chi-square test with one degree of freedom and an equal number of patients in each treatment group. Based on the estimated occurrence of the primary outcome measure of $15 \%$ in the SAVR group and $5 \%$ in the TAVI group during the first year, corresponding to an absolute risk reduction of $10 \%$ or a relative risk reduction of $66.7 \%$, and a chosen power of $1-\beta=80 \%$ and two-sided $\alpha=5 \%$, then 280 patients (140 patients per intervention group) are needed.

\section{Statistical analysis plan}

All analyses will be intention-to-treat analyses performed blinded with two-sided tests and 5\% level of significance. Table 2 shows for each outcome its priority (primary or to be used for explorative analyses), the time at which or the period during which it will be measured, the type of variable, and the analytical procedure (see below) to be used.

\section{Analytical procedures}

Depending on type of outcome measure, one of four types of regression analyses will be applied. The primary analytical results are those adjusted for age, trial site, presence of coronary disease not requiring revascularisation, and baseline value (possibly following multiple imputations; see below). Unadjusted analyses will also be carried out and major discrepancies between the results of adjusted and unadjusted analyses will be discussed. The types of regression analyses are as follows. 


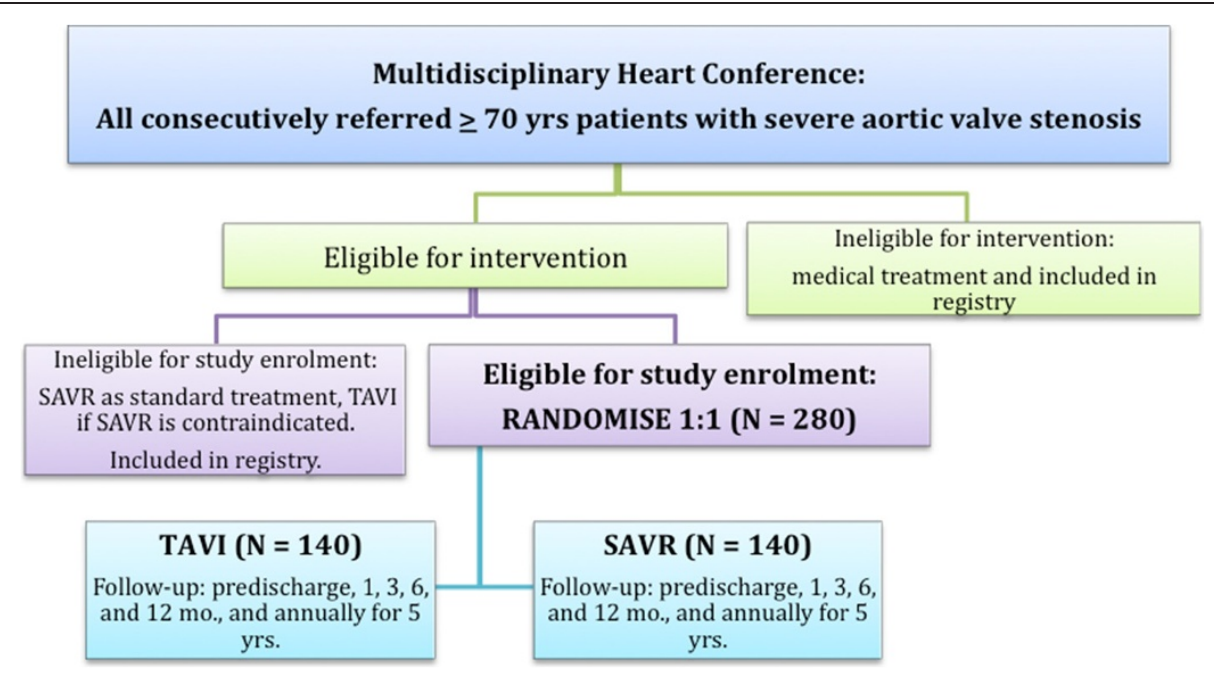

Figure 2 Study flow chart. An allcomers trial design. SAVR, surgical aortic valve replacement; TAVI, transcatheter aortic valve implantation.

Type 1: Logistic regression. In case of lack of convergence fit or non-estimable odds ratios, Fisher's exact test will be used and the protocol specified covariates thus disregarded.

Type 2: Includes a count of events within a specified period (12 months). Using the countreg procedure (SAS software version 9.3, SAS Institute Inc., Cary, NC, USA), the Poisson model, the negative binomial model, the zero inflated Poisson model, and the zero inflated negative binomial model will be compared by testing for overdispersion and comparing the average predicted count probabilities and the observed proportions. The best model will be used to analyse the count data. As a sensitivity analysis, a nonparametric test (Mann-Whitney test) will be conducted to compare the distributions of counts between the groups and the results will be discussed.

Type 3: Regression analysis using the proportional odds model for ordered variables. If the model assumption is significantly violated, then the Mann-Whitney test will be used.

Type 4: The general linear univariate model will be used. As a sensitivity analysis, a nonparametric test (MannWhitney test) will be conducted and the results discussed.

An explorative time-to-event analysis based on all available follow-up data on mortality will be conducted using Kaplan-Meier estimates and comparisons between groups with the use of the log-rank test.

\section{Missing values}

If the missingness exceeds $5 \%$ or the result of Little's test is significant $(P<0.05)$, then multiple imputations will be used (SPSS software version 17 or later, IBM Corp., Armonk, NY, USA). Ten imputed datasets will be generated and used.
The potential for bias caused by values missing not at random will be assessed for the primary outcome as a sensitivity analysis as follows: let A be the group with a beneficial effect of intervention as compared with the other group B. The missing values in A will be imputed by 1 (signifying that an event occurred) and those in group B by 0 .

\section{Multiplicity}

All outcomes apart from the primary outcome measure are exploratory and hypothesis generating.

The DMSC will conduct an interim analysis after the first 20 primary outcomes have occurred, which may prompt the DMSC to advise the steering committee to terminate or modify the trial if a significant difference is likely. The interim analyses are based on a statistical approach defined in the charter for the work of the DMSC. A random sample of $10 \%$ of patients will have their data monitored with source data verification by the regional good clinical practice unit. All statistical analysis will be performed with the use of SPSS or SAS software.

\section{Discussion}

The design of the NOTION trial is unique in that no randomised comparisons have been made between TAVI with the CoreValve System versus SAVR in a cohort of lowrisk, moderate-risk, and high-risk patients. The trial is designed to determine whether TAVI is safer than SAVR with similar efficacy in reducing AV stenosis. Favourable TAVI registry data are expanding, but randomised data are lacking. Despite this and high treatment costs, the number of TAVI procedures is increasing rapidly.

Designing a trial comparing TAVI versus SAVR is challenging. Complication types and their rates following 
Table 2 Outcomes, variable type, time/period of measurement, and statistical analysis for primary and exploratory outcomes

\begin{tabular}{|c|c|c|c|}
\hline Outcome $^{a}$ & Type of variable & Time or period of measurement & Statistical type of analysis ${ }^{\mathbf{b}}$ \\
\hline Myocardial infarction, stroke, or all-cause mortality (P) & Binary & During 12 months & 1 \\
\hline Myocardial infarction (E) & Binary & During 30 days. During 12 months & 1 \\
\hline Stroke $(E)$ & Binary & During 30 days. During 12 months & 1 \\
\hline All-cause mortality (E) & Binary & During 30 days. During 12 months & 1 \\
\hline Cardiovascular mortality (E) & Binary & During 30 days. During 12 months & 1 \\
\hline Prosthesis re-intervention (E) & Binary & During 30 days. During 12 months & 1 \\
\hline Conduction abnormality requiring pacemaker (E) & Binary & During 30 days. During 12 months & 1 \\
\hline Arrhythmia (E) & Binary & During 30 days. During 12 months & 1 \\
\hline Endocarditis (E) & Binary & During 30 days. During 12 months & 1 \\
\hline Mechanical ventilation in $\geq 24$ hours (E) & Binary & During 30 days & 1 \\
\hline Acute kidney injury (stage 2 or 3) (E) & Binary & During 30 days & 1 \\
\hline Access-site injury (major) (E) & Binary & During 30 days & 1 \\
\hline Bleeding (major-minor) (E) & Ordered & During 30 days & 3 \\
\hline Number of days hospitalised (E) & Discrete & During 12 months & 2 \\
\hline NYHA functional class (I to IV) (E) & Ordered $^{c}$ & At 3 months and at 12 months & 3 \\
\hline SF-36 Quality of life (E) & Continuous $^{c}$ & At 3 months and at 12 months & 4 \\
\hline Effective orifice area (E) & Continuous $^{c}$ & At 3 months and at 12 months & 4 \\
\hline Prosthesis peak gradient (E) & Continuous $^{\mathrm{c}}$ & At 3 months and at 12 months & 4 \\
\hline Prosthesis mean gradient (E) & Continuous $^{c}$ & At 3 months and at 12 months & 4 \\
\hline Prosthesis regurgitation (E) & Ordered $^{d}$ & At 3 months and at 12 months & 3 \\
\hline Prosthesis-patient mismatch (severe) (E) & Binary & At 3 months and at 12 months & 1 \\
\hline Left ventricular ejection fraction (E) & Continuous $^{c}$ & At 3 months and at 12 months & 4 \\
\hline
\end{tabular}

P, primary outcome; E, exploratory outcome; NYHA, New York Heart Association; SF-36, Short-form health survey with 36 questions. ${ }^{2}$ Outcomes defined according to Valve Academic Research Consortium criteria. ${ }^{b}$ See analytical procedures under Statistical analysis plan. ${ }^{c}$ Baseline value is measured and is included in the adjusted analysis. ${ }^{\mathrm{d}}$ Graded as none, mild, moderate, or severe.

endovascular and surgical procedures are different and must be weighed quantitatively as well as qualitatively. There was very little experience with TAVI in low-risk and moderate-risk patients and no randomised trials involving TAVI at the time of the design of this trial during 2008/09, so the expected rates of outcome measures are therefore based on clinical judgement, in-hospital complication databases, registry data, STS score, and EuroSCORE. Consequently, there is a degree of uncertainty in estimating the trial sample size as well as calculating power for the exploratory outcomes.

Of particular concern during and after TAVI is the possible elevated risk of stroke, unknown durability of the prosthesis, and the long-term cardiac effect of the frequent paravalvular leakage and conduction abnormalities. High-risk patients have clinical characteristics (for example, previous stroke, peripheral vascular disease, hypertension) that increase the risk of stroke and other complications with any major cardiovascular intervention. Less morbid patients with a good functional preintervention status are likely to benefit even more from TAVI than these high-risk patients, and complication rates are expected to decline. However, no safety data exist for this particular patient population. The decision to include this low-risk group of patients in the trial therefore presents a dilemma, since balancing the risk of the abovementioned complications and expanding our knowledge of this new treatment modality is difficult. The setting of a randomised clinical trial with extensive follow-up and data safety monitoring seems to justify the decision. Should the prosthesis used in either group become dysfunctional, most patients will be candidates for either re-TAVI (valve-in-valve procedure) or surgical prosthesis replacement $[21,33,34]$.

Patient eligibility for AV intervention and trial enrolment is evaluated consecutively at a multidisciplinary conference. In an attempt to explore the effect of TAVI in AV stenosis most specifically, patients with significant coronary artery disease requiring revascularisation at the time of referral are excluded. Study results will consequently not be readily applicable for a large group of AV stenosis patients requiring concomitant revascularisation. On the contrary, the study cohort should reflect patients seen in clinical practice, and therefore patients 
with previous, but not recent, myocardial or cerebral infarction are included. Patients with myocardial infarction and/or percutaneous coronary intervention with a drugeluted stent within the previous year are excluded, however, since discontinuation of dual-antiplatelet therapy prior to surgery could expose these patients to a thromboembolic risk. Patients with atrial fibrillation are also included. To avoid redo-valve procedures due to expected prosthesis degeneration and mechanical valve prostheses, we chose the 70-year-old minimum age criterion.

Patients are excluded if they have undergone previous open cardiac surgery, since SAVR with re-sternotomy and mediastinal dissection after pericardiotomy represents a different and higher risk procedure than firsttime SAVR.

Similar postprocedure antithrombotic therapy in both treatment groups was chosen to avoid the confounding effect of different medical antithromboembolic protection in the two intervention groups. This strategy was not in accordance with the guidelines at the time of designing the trial, which recommended 3 months of oral anticoagulation after SAVR [4]. No evidence existed for the optimal antithrombotic treatment after TAVI [35]. This situation has not changed substantially [5].

Only one TAVI system is used in the trial. Most centres use only one system as the technology is still new and requires a substantial amount of experience to be performed safely. However, as experience with more devices grows, a more complete picture of TAVI and its effectiveness should be evaluated in randomised trials comparing surgery with TAVI using all available delivery systems and prostheses.

We have tried to minimise bias in different ways. Selection bias is minimised by consecutive enrolment and central randomisation ensuring random allocation and allocation concealment [36,37]. The trial is not blinded owing to the inherently different nature of the two treatment modalities. Unblinded trials are at risk of introducing performance, collateral intervention, attrition, and assessment bias [36,37]. No investigator performs both interventions, but obvious differences in experience with a new procedure (TAVI) and a well-established procedure (SAVR) will introduce performance bias. Investigators performing TAVI have all done more than 75 procedures before this trial began. All cardiac surgeon investigators have extensive SAVR experience. The blinding of the event adjudication process for the primary outcome will diminish assessment bias. Publication of the trial protocol prior to analysing and reporting the data will prevent outcome reporting bias, and the multicentre design will improve external validity. The trial budget will unfortunately only accommodate $10 \%$ data monitoring, which obviously presents a limitation. Members of the independent DMSC and event adjudication committee have not been involved in any aspects of the trial design or conduct.

Other trials comparing new endovascular treatments against surgery have demonstrated that the overall benefit of a new procedure requires weighing of the relative risks and benefits in specific patient populations. There must be a reasonable balance between safety, effectiveness, and device durability. Safety may be more important than suboptimal effectiveness and durability for some patients. This concept demands patient assessment by an interventionalist and a cardiac surgeon before guidance can be given to the patient. Trials have thus suggested that surgery could perhaps be deferred as first-line treatment for certain high-operative-risk patients in myocardial revascularisation and mitral valve repair. This suggestion is also in accordance with patient preferences and wishes toward less invasive treatment options.

In conclusion, the NOTION trial is designed to compare the safety and effectiveness of TAVI versus conventional SAVR in the treatment of patients 70 years of age or older with stand-alone severe degenerative AV stenosis. TAVI may reduce postoperative morbidity and mortality without compromising a favourable functional outcome. This could expand the patient population referred for AV intervention and change the type of intervention used.

\section{Trial status}

Since screening began in December 2009 and up to December 2012, 269 patients have been enrolled. Enrolment is expected to be complete at the end of January 2013.

\section{Abbreviations}

AV: aortic valve; DMSC: data monitoring and safety committee; ECG: electrocardiogram; EuroSCORE: European System for Cardiac Operative Risk Evaluation; NOTION: Nordic Aortic Valve Intervention; SAVR: surgical aortic valve replacement; STS: Society of Thoracic Surgery Predicted Risk of Mortality; TAVI: transcatheter aortic valve implantation.

\section{Competing interests}

Principal investigator LS serves as a physician proctor for Medtronic Inc. This role implies supervising other centres in implementing the TAVI device and implantation system. LS has received consulting and lecture fees, grant support, and reimbursement for travel expenses from Medtronic Inc. All remaining authors declare that they have no competing interests.

\section{Authors' contributions}

Authors HGT, LS, and DAS conceived and designed the study, acquired data, and drafted the manuscript. All other authors acquired data and/or made substantial contributions and revisions to the manuscript. All authors read and approved the final manuscript.

\section{Acknowledgements}

The authors acknowledge the Danish Heart Foundation and all its beneficiaries for financial support. They thank Dr Peter Clemmensen, Dr Gorm Boje Jensen, and Dr Ulla Davidsen at the Danish Heart Foundation, Dr Bo Juel Kjeldsen at Odense University Hospital, Denmark, Dr Andreas Wallinder, Dr Truls Råmunddal, and Dr Petur Petursson at Sahlgrenska University Hospital, Sweden, research nurses Line M Kristensen and Lisette L Larsen, and all patients participating in the trial. 


\section{Author details}

${ }^{1}$ Department of Cardiothoracic Surgery, The Heart Centre, Rigshospitalet, Copenhagen University Hospital, Blegdamsvej 9, DK 2100, Copenhagen, Denmark. ${ }^{2}$ Department of Cardiology, The Heart Centre, Rigshospitalet, Copenhagen University Hospital, Blegdamsvej 9, DK 2100, Copenhagen, Denmark. ${ }^{3}$ Department of Cardiac Anesthesia, The Heart Centre, Rigshospitalet, Copenhagen University Hospital, Blegdamsvej 9, DK 2100, Copenhagen, Denmark. ${ }^{4}$ Department of Cardiology, Odense University Hospital, Sdr. Boulevard 29, DK 5000, Odense, Denmark. ${ }^{5}$ Copenhagen Trial Unit, Centre for Clinical Intervention Research, Rigshospitalet, Copenhagen University Hospital, Tagensvej 22, DK 2100, Copenhagen, Denmark.

Received: 14 May 2012 Accepted: 18 December 2012 Published: 9 January 2013

\section{References}

1. lung B, Baron G, Butchart EG, Delahaye F, Gohlke-Bärwolf C, Levang OW, Tornos P, Vanoverschelde $J$, Vermeer F, Boersma E, Ravaud P, Vahanian A: A prospective survey of patients with valvular heart disease in Europe: the Euro Heart Survey on Valvular Heart Disease. Eur Heart J 2003, 24:1231-1243.

2. Nkomo VT, Gardin JM, Skelton TN, Gottdiener JS, Scott CG, Enriquez-Sarano M: Burden of valvular heart diseases: a population-based study. Lancet 2006, 368:1005-1011.

3. Ross J Jr, Braunwald E: Aortic stenosis. Circulation 1968, 38:61-67.

4. Vahanian A, Baumgartner H, Bax J, Butchart E, Dion R, Filippatos G, Flachskampf F, Hall R, lung B, Kasprzak J, Nataf P, Tornos P, Torracca L, Wenink A, Task Force on the Management of Valvular Hearth Disease of the European Society of Cardiology, ESC Committee for Practice Guidelines: Guidelines on the management of valvular heart disease: the task force on the management of valvular heart disease of the European Society of cardiology. Eur Heart J 2007, 28:230-268.

5. Vahanian A, lung B: The new ESC/EACTS guidelines on the management of valvular heart disease. Arch CardiovasC Dis 2012, 105:465-467.

6. lung B, Cachier A, Baron G, Messika-Zeitoun D, Delahaye F, Tornos P Gohlke-Bärwolf C, Boersma E, Ravaud P, Vahanian A: Decision-making in elderly patients with severe aortic stenosis: why are so many denied surgery? Eur Heart J 2005, 26:2714-2720.

7. O'Brien SM, Shahian DM, Filardo G, Ferraris VA, Haan CK, Rich JB, Normand SL, DeLong ER, Shewan CM, Dokholyan RS, Peterson ED, Edwards FH, Anderson RP, Society of Thoracic Surgeons Quality Measurement Task Force: The Society of Thoracic Surgeons 2008 cardiac surgery risk models: part 2-isolated valve surgery. Ann Thorac Surg 2009, 88:S23-\$42.

8. Roques F, Nashef SA, Michel P: Risk factors for early mortality after valve surgery in Europe in the 1990s: lessons from the EuroSCORE pilot program. J Heart Valve Dis 2001, 10:572-577. discussion 577-578.

9. Nashef SA, Roques F, Sharples LD, Nilsson J, Smith C, Goldstone AR, Lockowandt U: EuroSCORE II. Eur J Cardiothorac Surg 2012, 41:734-744. discussion 744-735.

10. Andersen $H R$, Knudsen $L L$, Hasenkam JM: Transluminal implantation of artificial heart valves. Description of a new expandable aortic valve and initial results with implantation by catheter technique in closed chest pigs. Eur Heart J 1992, 13:704-708.

11. Cribier A, Eltchaninoff H, Bash A, Borenstein N, Tron C, Bauer F, Derumeaux $G$, Anselme F, Laborde F, Leon MB: Percutaneous transcatheter implantation of an aortic valve prosthesis for calcific aortic stenosis: first human case description. Circulation 2002, 106:3006-3008.

12. Cribier A, Eltchaninoff $H$, Tron C, Bauer F, Agatiello C, Sebagh L, Bash A, Nusimovici D, Litzler PY, Bessou JP, Leon MB: Early experience with percutaneous transcatheter implantation of heart valve prosthesis for the treatment of end-stage inoperable patients with calcific aortic stenosis. J Am Coll Cardiol 2004, 43:698-703.

13. Grube E, Laborde JC, Gerckens U, Felderhoff T, Sauren B, Buellesfeld L, Mueller R, Menichelli M, Schmidt T, Zickmann B, Iversen S, Stone GW: Percutaneous implantation of the CoreValve self-expanding valve prosthesis in high-risk patients with aortic valve disease: the Siegburg first-in-man study. Circulation 2006, 114:1616-1624.

14. Kahlert P, Knipp SC, Schlamann M, Thielmann M, Al-Rashid F, Weber M, Johansson U, Wendt D, Jakob HG, Forsting M, Sack S, Erbel R, Eggebrecht H: Silent and apparent cerebral ischemia after percutaneous transfemoral aortic valve implantation: a diffusion-weighted magnetic resonance imaging study. Circulation 2010, 121:870-878.

15. Gilard $M$, Eltchaninoff $H$, lung B, Donzeau-Gouge $P$, Chevreul $K$, Fajadet J, Leprince $P$, Leguerrier A, Lievre M, Prat A, Teiger E, Lefevre T, Himbert D, Tchetche D, Carrié D, Albat B, Cribier A, Rioufol G, Sudre A, Blanchard D, Collet F, Dos Santos P, Meneveau N, Tirouvanziam A, Caussin C, Guyon P, Boschat J, Le Breton H, Collart F, Houel R, et al: Registry of transcatheter aortic-valve implantation in high-risk patients. N Engl J Med 2012, 366:1705-1715.

16. Webb J, Cribier A: Percutaneous transarterial aortic valve implantation: what do we know? Eur Heart J 2011, 32:140-147.

17. Tzikas A, van Dalen BM, Van Mieghem NM, Gutierrez-Chico JL, Nuis RJ, Kauer F, Schultz C, Serruys PW, de Jaegere PP, Geleijnse ML: Frequency of conduction abnormalities after transcatheter aortic valve implantation with the Medtronic-CoreValve and the effect on left ventricular ejection fraction. Am J Cardiol 2011, 107:285-289.

18. Olsen LK, Engstrøm T, Wachtell K, Kristensen T, Hassager C, Ihlemann N, Møller JE, Andersen LW, Olsen PS, Søndergaard L: Percutaneous aortic valve replacement - initial experience and results. Ugeskr Laeger 2009, 171:1289-1293.

19. Moat $N E$, Ludman $P$, de Belder MA, Bridgewater $B$, Cunningham AD, Young CP, Thomas M, Kovac J, Spyt T, MacCarthy PA, Wendler O, Hildick-Smith D, Davies SW, Trivedi U, Blackman DJ, Levy RD, Brecker SJ, Baumbach A, Daniel $\mathrm{T}$, Gray $H$, Mullen MJ: Long-term outcomes after transcatheter aortic valve implantation in high-risk patients with severe aortic stenosis: The U.K. TAVI (United Kingdom Transcatheter Aortic Valve Implantation) registry. J Am Coll Cardiol 2011, 58:2130-2138.

20. Grube E, Schuler G, Buellesfeld L, Gerckens U, Linke A, Wenaweser P, Sauren B, Mohr FW, Walther T, Zickmann B, Iversen S, Felderhoff T, Cartier R, Bonan $\mathrm{R}$ : Percutaneous aortic valve replacement for severe aortic stenosis in high-risk patients using the second- and current third-generation selfexpanding CoreValve prosthesis: device success and 30-day clinical outcome. J Am Coll Cardiol 2007, 50:69-76.

21. Hammerstingl C, Nickenig G, Grube E: Treatment of a degenerative stenosed CoreValve ${ }^{\circledR}$ aortic bioprosthesis by transcatheter valve-in-valve insertion. Catheter Cardiovasc Interv 2012, 79:748-755.

22. Leon MB, Smith CR, Mack M, Miller DC, Moses JW, Svensson LG, Tuzcu EM, Webb JG, Fontana GP, Makkar RR, Brown DL, Block PC, Guyton RA, Pichard AD, Bavaria JE, Herrmann HC, Douglas PS, Petersen JL, Akin JJ, Anderson WN, Wang D, Pocock S, PARTNER Trial Investigators: Transcatheter aorticvalve implantation for aortic stenosis in patients who cannot undergo surgery. N Engl J Med 2010, 363:1597-1607.

23. Smith CR, Leon MB, Mack MJ, Miller DC, Moses JW, Svensson LG, Tuzcu EM, Webb JG, Fontana GP, Makkar RR, Williams M, Dewey T, Kapadia S, Babaliaros V, Thourani VH, Corso P, Pichard AD, Bavaria JE, Herrmann HC, Akin JJ, Anderson WN, Wang D, Pocock SJ, PARTNER Trial Investigators: Transcatheter versus surgical aortic-valve replacement in high-risk patients. N Engl J Med 2011, 364:2187-2198.

24. Makkar RR, Fontana GP, Jilaihawi $H$, Kapadia S, Pichard AD, Douglas PS, Thourani VH, Babaliaros VC, Webb JG, Herrmann HC, Bavaria JE, Kodali S, Brown DL, Bowers B, Dewey TM, Svensson LG, Tuzcu M, Moses JW, Williams MR, Siegel RJ, Akin JJ, Anderson WN, Pocock S, Smith CR, Leon MB, PARTNER Trial Investigators: Transcatheter aortic-valve replacement for inoperable severe aortic stenosis. N Engl J Med 2012, 366:1696-1704.

25. Kodali SK, Williams MR, Smith CR, Svensson LG, Webb JG, Makkar RR, Fontana GP, Dewey TM, Thourani VH, Pichard AD, Fischbein M, Szeto WY, Lim S, Greason KL, Teirstein PS, Malaisrie SC, Douglas PS, Hahn RT, Whisenant B, Zajarias A, Wang D, Akin JJ, Anderson WN, Leon MB, PARTNER Trial Investigators: Two-year outcomes after transcatheter or surgical aortic-valve replacement. N Engl J Med 2012, 366:1686-1695.

26. Nielsen HH, Klaaborg KE, Nissen H, Terp K, Mortensen PE, Kjeldsen BJ, Jakobsen CJ, Andersen HR, Egeblad H, Krusell LR, Thuesen L, Hjortdal VE: A prospective, randomised trial of transapical transcatheter aortic valve implantation vs. surgical aortic valve replacement in operable elderly patients with aortic stenosis: the STACCATO trial. Eurolntervention 2012, 8:383-389.

27. Vahanian A, Alfieri OR, Al-Attar N, Antunes MJ, Bax J, Cormier B, Cribier A De Jaegere P, Fournial G, Kappetein AP, Kovac J, Ludgate S, Maisano F, Moat N, Mohr FW, Nataf P, Pierard L, Pomar JL, Schofer J, Tornos P, Tuzcu $M$, van Hout $B$, von Segesser LK, Walther T: Transcatheter valve implantation for patients with aortic stenosis: a position statement from 
the European Association of Cardio-Thoracic Surgery (EACTS) and the European Society of Cardiology (ESC), in collaboration with the European Association of Percutaneous Cardiovascular Interventions (EAPCI). Eur J Cardiothorac Surg 2008, 34:1-8.

28. Andersen TF, Madsen M, Jørgensen J, Mellemkjoer L, Olsen JH: The Danish National Hospital Register. A valuable source of data for modern health sciences. Dan Med Bull 1999, 46:263-268.

29. Pedersen CB, Gøtzsche H, Møller JO, Mortensen PB: The Danish Civil Registration System. A cohort of eight million persons. Dan Med Bull 2006, 53:441-449.

30. Helweg-Larsen K: The Danish register of causes of death. Scand J Public Health 2011, 39:26-29.

31. Leon MB, Piazza N, Nikolsky E, Blackstone EH, Cutlip DE, Kappetein AP, Krucoff MW, Mack M, Mehran R, Miller C, Morel MA, Petersen J, Popma JJ, Takkenberg JJ, Vahanian A, van Es GA, Vranckx P, Webb JG, Windecker S, Serruys PW: Standardized endpoint definitions for transcatheter aortic valve implantation clinical trials: a consensus report from the Valve Academic Research Consortium. Eur Heart J 2011, 32:205-217.

32. Kappetein AP, Head SJ, Généreux P, Piazza N, van Mieghem NM, Blackstone EH, Brott TG, Cohen DJ, Cutlip DE, van Es GA, Hahn RT, Kirtane AJ, Krucoff MW, Kodali S, Mack MJ, Mehran R, Rodés-Cabau J, Vranckx P, Webb JG, Windecker S, Serruys PW, Leon MB: Updated standardized endpoint definitions for transcatheter aortic valve implantation: the Valve Academic Research Consortium-2 consensus document (VARC-2). Eur J Cardiothorac Surg 2012, 42:S45-S60.

33. Dvir D, Webb J, Brecker S, Bleiziffer S, Hildick-Smith D, Colombo A, Descoutures F, Hengstenberg C, Moat NE, Bekeredjian R, Napodano M, Testa L, Lefevre T, Guetta V, Nissen H, Hernández JM, Roy D, Teles RC, Segev A, Dumonteil N, Fiorina C, Gotzmann M, Tchetche D, Abdel-Wahab M, De Marco F, Baumbach A, Laborde JC, Kornowski R: Transcatheter aortic valve replacement for degenerative bioprosthetic surgical valves: results from the global valve-in-valve registry. Circulation 2012, 126:2335-2344.

34. Thyregod HG, Lund JT, Engstrøm T, Steinbrüchel DA: Transcatheter aortic valve prosthesis surgically replaced 4 months after implantation. Eur J Cardiothorac Surg 2010, 37:494-496.

35. Ussia GP, Scarabelli M, Mulè M, Barbanti M, Sarkar K, Cammalleri V, Immè S, Aruta P, Pistritto AM, Gulino S, Deste W, Capodanno D, Tamburino C: Dual antiplatelet therapy versus aspirin alone in patients undergoing transcatheter aortic valve implantation. Am J Cardio/ 2011, 108:1772-1776.

36. Wood L, Egger M, Gluud LL, Schulz KF, Jüni P, Altman DG, Gluud C, Martin RM, Wood AJ, Sterne JA: Empirical evidence of bias in treatment effect estimates in controlled trials with different interventions and outcomes: meta-epidemiological study. BMJ 2008, 336:601-605.

37. Savovic J, Jones HE, Altman DG, Altman DG, Harris RJ, Jüni P, Pildal J, AlsNielsen B, Balk EM, Gluud C, Gluud LL, loannidis JP, Schulz KF, Beynon R, Welton NJ, Wood L, Moher D, Deeks JJ, Sterne JA: Influence of reported study design characteristics on intervention effect estimates from randomized, controlled trials. Ann Intern Med 2012, 157:429-438.

doi:10.1186/1745-6215-14-11

Cite this article as: Thyregod et al:: The Nordic Aortic Valve Intervention (NOTION) trial comparing transcatheter versus surgical valve implantation: study protocol for a randomised controlled trial. Trials 2013 14:11.

\section{Submit your next manuscript to BioMed Central and take full advantage of:}

- Convenient online submission

- Thorough peer review

- No space constraints or color figure charges

- Immediate publication on acceptance

- Inclusion in PubMed, CAS, Scopus and Google Scholar

- Research which is freely available for redistribution 